\title{
Dysfunction of outer segment guanylate cyclase caused by retinal disease related mutations
}

\author{
Patrick Zägel ${ }^{1}$ and Karl-Wilhelm Koch ${ }^{1,2} *$ \\ ' Biochemistry Group, Department of Neurosciences, Carl von Ossietzky University Oldenburg, Oldenburg, Germany \\ ${ }^{2}$ Research Center Neurosensory Science, Carl von Ossietzky University Oldenburg, Oldenburg, Germany
}

\section{Edited by:}

Rameshwar K. Sharma, Salus

University, USA

\section{Reviewed by:}

Rameshwar K. Sharma, Salus

University, USA

Teresa Duda, Salus University, USA

\section{*Correspondence:}

Karl-Wilhelm Koch, Biochemistry Group, Department of

Neurosciences, Carl von Ossietzky

University Oldenburg, Oldenburg

D-26111, Germany

e-mail:karl.w.koch@uni-oldenburg.de
Membrane bound guanylate cyclases are expressed in rod and cone cells of the vertebrate retina and mutations in several domains of rod outer segment guanylate cyclase 1 (ROSGC1 encoded by the gene GUCY2D) correlate with different forms of retinal degenerations. In the present work we investigated the biochemical consequences of three point mutations, one is located in position P575L in the juxtamembrane domain close to the kinase homology domain and two are located in the cyclase catalytic domain at H1019P and $\mathrm{P} 1069 \mathrm{R}$. These mutations correlate with various retinal diseases like autosomal dominant progressive cone degeneration, e.g., Leber Congenital Amaurosis and a juvenile form of retinitis pigmentosa. Wildtype and mutant forms of ROS-GC1 were heterologously expressed in HEK cells, their cellular distribution was investigated and activity profiles in the presence and absence of guanylate cyclase-activating proteins were measured. The mutant P575L was active under all tested conditions, but it displayed a twofold shift in the $\mathrm{Ca}^{2+}$-sensitivity, whereas the mutant P1069R remained inactive despite normal expression levels. The mutation H1019P caused the cyclase to become more labile. The different biochemical consequences of these mutations seem to reflect the different clinical symptoms. The mutation P575L induces a dysregulation of the $\mathrm{Ca}^{2+}$-sensitive cyclase activation profile causing a slow progression of the disease by the distortion of the $\mathrm{Ca}^{2+}{ }_{-} \mathrm{cGMP}$ homeostasis. In contrast, a strong reduction in cGMP synthesis due to an inactive or structurally unstable ROS-GC1 would trigger more severe forms of retinal diseases.

Keywords: photoreceptor guanylate cyclase, GCAP, retinal dystrophy, neuronal calcium sensor, phototransduction

\section{INTRODUCTION}

Inherited retinal diseases are caused by a heterogeneous group of mutations in retinal genes (Perrault etal., 2000; den Hollander et al., 2008; Athanasiou et al., 2013). Among them are a large number of genes coding for proteins involved in phototransduction processes in the outer segments of rods and cones. Genetic heterogeneity is paralleled by diverse phenotypical and clinical characteristics of retinal degenerations, which are classified in disorders like cone-rod dystrophies, retinitis pigmentosa, and leber congenital amaurosis (LCA). These disease forms lead to a loss of visual function, but they differ in the progression of specific visual impairments.

Phototransduction in rods and cones is centered on the lighttriggered hydrolysis of the second messenger cyclic GMP, its powerful re-synthesis after illumination and its role as a ligand targeting a cyclic nucleotide-gated channel in the plasma membrane (Pugh and Lamb, 2000; Kaupp and Seifert, 2002; Luo et al., 2008). Phototransduction is further under control of several negative feedback loops that are important for recovery of the photoresponse from illumination and for adjusting the cell performance to ambient background light (Fain et al., 2001). One crucial molecular factor involved in the regulation of phototransduction is $\mathrm{Ca}^{2+}$, which binds to and dissociates from $\mathrm{Ca}^{2+}$-sensor proteins under oscillating changes in cytoplasmic $\mathrm{Ca}^{2+}$-concentration $\left[\mathrm{Ca}^{2+}\right]$.
A multi-protein complex operating at the interface between changing concentrations of cGMP and $\left[\mathrm{Ca}^{2+}\right]$ consists of a sensory type membrane-bound guanylate cyclase that is regulated by guanylate cyclase-activating proteins (GCAPs; Stephen et al., 2008; Dizhoor et al., 2010; Koch et al., 2010; Koch and Dell'Orco, 2013). So far, mutations in patients suffering from retinal diseases are found in the gene GUCA1A encoding the $\mathrm{Ca}^{2+}$-sensor GCAP1 and GUCY2D coding for the outer segment guanylate cyclase type 1 (ROS-GC1) (Kitiratschky et al., 2008; Behnen et al., 2010; Hunt et al., 2010). Previous research was focused on elucidating the molecular causes and cellular consequences of point mutations in ROS-GC1 and GCAP1 and in a further step to test therapeutic strategies involving RNA interference techniques and transgenic mouse lines (Buch et al., 2011; Jiang et al., 2011; Boye et al., 2013).

However, disease causing mutations can also point to critical amino acid positions that determine structure-function relationships in natively folded protein structures and therefore might help to gain mechanistic insights of protein function and regulation (Duda et al., 1999a, 2000; Ramamurthy et al., 2001). For example, a biochemical study on mutations in the so-called dimerization domain of ROS-GC1 correlating with cone-rod dystrophy recently revealed that this dimerization or linker domain operates as a $\mathrm{Ca}^{2+}$-sensitive control switch module (Zägel et al., 2013). 
In the present work we tested the biochemical consequences of three point mutations (Figure 1). The mutation P575L was found in a family, where clinically affected members suffer from progressive cone degeneration (Small et al., 2008). The amino acid substitution is located in the juxtamembrane domain (JMD), which is a specific region of sensory GCs upstream of the kinase homology domain (KHD). H1019P is located in the cyclase catalytic domain (CCD) and patients with this missense mutation show the typical clinical symptoms of LCA type 1 suffering from severe visual impairment at birth (Perrault et al., 2000). Finally, P1069R is also within the CCD and a patient carrying this mutation was diagnosed with juvenile retinitis pigmentosa (Booji et al., 2005).

So far, none of these mutations have been investigated on a molecular level. Two of the main questions are for example what the consequences on guanylate cyclase activity are and which $\mathrm{Ca}^{2+}$-dependent activation profiles can be observed in the presence of GCAPs. These topics are addressed in the present contribution.

\section{MATERIALS AND METHODS CLONING AND SITE-DIRECTED MUTAGENESIS OF HUMAN ROS-GC1 CONSTRUCTS}

The cDNA of human ROS-GC1 was cloned into the vector pIRES2-eGFP (Clonetech) exactly as described previously (Zägel etal., 2013). DNA was prepared by using the DNA purification kit of Promega according to manufacturer's instruction. The mutant P575L was produced by using the following primers for PCR amplification: 5'-GCTATCCGCCTAGCAACCAAGACG$3^{\prime}$ (forward) and 5'-CGTCTTGGTTGCTAGGCGGATAGC-3' (reverse). Site-directed mutagenesis yielding the mutants H1019P and P1069R was achieved by performing two PCRs each using the ROS-GC1-WT gene in the pIRES2-eGFP vector as template. Primers for the PCR were for creating H1019P: 5'-CCTTACCGCATCCCCGTGAACTTGAG-3' and 5'-TCAAGAGAACTGGCCCGGCCGC-3' (forward and reverse primer, first PCR) and 5'-AAAGCTAGCCACCATGACCGCCTGCGCCCGCC-3' and 5'-CTCAAGTTCACGGGGATGCGGTAAGG-3' (forward and reverse primer, second PCR). P1069R was obtained with $5^{\prime}$-CCCATCCCCAAACGGCCTGACCTG- $3^{\prime}$ and 5'-TCAAGAGAACTGGCCCGGCCGC-3' (forward and reverse primer in the first PCR) and $5^{\prime}$-AAAGCTAGCCACCATGACCGC CTGCGCCCGCC-3' and 5'-CAGGTCAGGCCGTTTGGGGATGGG-3' (forward and reverse primer in the second PCR). The resulting two fragments were used in a further PCR to obtain a complete mutated ROS-GC1-DNA construct (3320 bp) by employing the primers 5' $5^{\prime}$ AAAGCTAGCCACCATGACCGCCTGCGCCCGCC-3' and 5'-TCAAGAGAACTGGCCCGGCCGC-3'. The final construct was cut with Nhe I, phosphorylated with T4 polynucleotide kinase and ligated in the pIRES2-EGFP vector that was cut by Nhe I/Sma I. Clone products were verified by sequencing.

\section{HETEROLOGOUS EXPRESSION OF ROS-GC1 AND GCAPS}

WT and mutant forms of ROS-GC1 were expressed in HEK flip 293 cells. For this purpose cells were cultivated in minimal essential medium under standard conditions as described

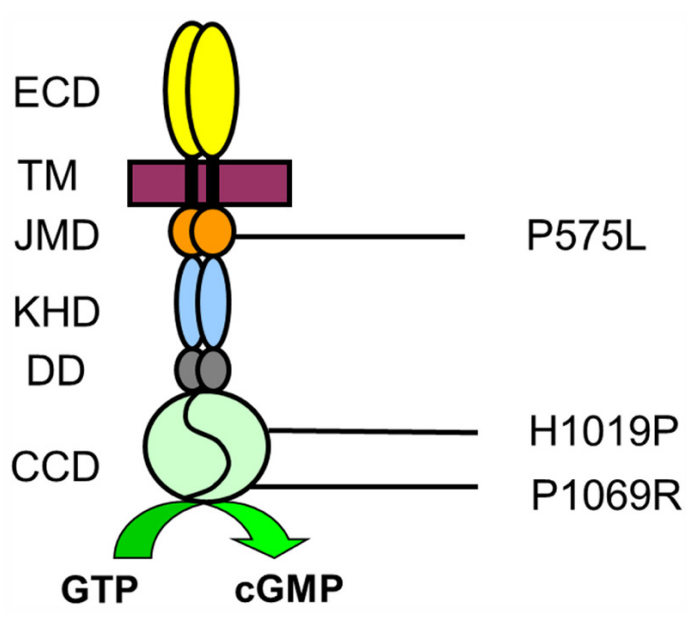

FIGURE 1 | Amino acid positions of mutations in ROS-GC1. Topology of ROS-GC1 domains is indicated as extracellular domain (ECD) corresponding to the intradiscal region when cyclases are expressed in rod outer segments, transmembrane domain (TM), JMD, KHD, dimerization domain, and cyclase catalytic domain (CCD). Numbers correspond to the unprocessed human sequence.

(Koch and Helten, 2008). After culturing 3-5 $\times 10^{6}$ cells were harvested in $100 \mu \mathrm{L}$ of a buffer containing $5 \mathrm{mM} \mathrm{KCl}, 20 \mathrm{mM}$ $\mathrm{MgCl}_{2}$, and $150 \mathrm{mM}$ phosphate buffer $\mathrm{pH}$ 7.2. For transfection, $5 \mu \mathrm{g}$ of DNA was added and cells were electroporated with the CLB system (Lonza). Further treatment of cells and harvesting for subsequent guanylate cyclase assays was done exactly as described (Zägel et al., 2013). GCAP1 and GCAP2 were expressed in E. coli and purified to homogeneity by anion exchange and size exclusion chromatography as previously reported (Hwang et al., 2003; Koch and Helten, 2008). Myristoylation of GCAPs during bacterial expression was accomplished by co-transforming E. coli cells with $N$-myristoyl-transferase from yeast and supplementation with myristic acid. To ensure a high degree of myristoylation of GCAP1, which lacks the consensus site for yeast $N$-myristoyl-transferase, we used the $D^{6}$ S-mutant of GCAP1 (Krylov et al., 1999; Hwang etal., 2003). The degree of myristoylation of both GCAPs was typically higher than $90 \%$ as checked by using an analytical HPLC run on a reverse-phase column.

\section{GUANYLATE CYCLASE ASSAY}

Recombinant human ROS-GC1 in HEK cell membranes was measured as described before (Koch and Helten, 2008; Zägel et al., 2013). In addition to measuring the basal guanylate cyclase activity we mainly tested two effects of GCAPs on the activation profile of the cyclase. In a first set we added purified bovine GCAP1 or GCAP2 at saturating $10 \mu \mathrm{M}$ to washed HEK cell membranes and incubated samples at different free $\left[\mathrm{Ca}^{2+}\right]$ using a $\mathrm{Ca}^{2+}$-EGTA buffer system. Details of the procedure have been described in several previous publications (Hwang et al., 2003; Koch and Helten, 2008; Zägel et al., 2013). Data were evaluated to obtain the $\left[\mathrm{Ca}^{2+}\right]$, at which the activation is half-maximal $\left(\mathrm{IC}_{50}\right)$. In a second set of guanylate cyclase 
assays we incubated recombinant ROS-GC1 with increasing concentrations of GCAP1 and GCAP2 $(0-20 \mu \mathrm{M})$ for $30 \mathrm{~min}$ under $\mathrm{Ca}^{2+}$-free conditions. GCAPs were liganded with $\mathrm{Mg}^{2+}$ (Peshenko and Dizhoor, 2004) using the same buffer composition as described (Koch and Helten, 2008). The latter activity measurements yielded apparent affinities ( $\mathrm{EC}_{50}$-values) of GCAPs for WT and mutant ROS-GC1. Activity values were obtained from at least 3-4 different data sets and were used to calculate the mean \pm standard deviation (SD). A student's $t$-test was performed to check for significant differences between two sets of data.

\section{IMMUNOHISTOCHEMISTRY}

Localization of ROS-GC1 WT and mutants in HEK cells by immunofluorescence microscopy was done as described before using an Olympus fluorescence microscope (Zägel et al., 2013). The following primary antibodies were used for detection: antiROS-GC1 (1:100; ROS-GC1 (H-225), sc50512, rabbit polyclonal IgG; Santa Cruz Biotechnology), anti-Na ${ }^{+} / \mathrm{K}^{+}$-ATPase (1:200; $\mathrm{Na}^{+} / \mathrm{K}^{+}$-ATPase, a (H-3), sc-48345, mouse monoclonal $\mathrm{IgG}_{2 \mathrm{~b}}$; Santa Cruz Biotechnology), and anti-calnexin [1:200; calnexin (E-10), sc-46669, mouse monoclonal IgG $2 \mathrm{a}$; Santa Cruz Biotechnology]. Secondary antibodies were donkey anti-rabbit conjugated to Fura350 (dilution 1:200) from Invitrogen and goat anti-mouse conjugated with Dylight594 from Thermo Scientific, USA used in a dilution of 1:500. Incubation and washing buffers were exactly as described (Zägel et al., 2013).

\section{RESULTS AND DISCUSSION}

\section{LOCALIZATION OF ROS-GC1 CONSTRUCTS}

Previous work has shown that ROS-GC1 can be expressed heterologously in HEK293 cells in sufficient quantity to allow subsequent biochemical studies (Hwang et al., 2003; Koch and Helten, 2008; Zägel et al., 2013). In HEK293 cells the enzyme was found to be present in cell membranes and mainly co-localized with the endoplasmic reticulum. Probing the cells with an anti-ROS-GC1 antibody (Figure 2A, middle panel) and using specific markers for the plasma membrane (anti-Na $\mathrm{Na}^{+} / \mathrm{K}^{+}$-ATPase antibody, top left panel in Figure 2A) and for the endoplasmic reticulum (anticalnexin, top right panel) we obtained a staining pattern in the overlay image (Figure 2A, bottom panels) in agreement with published results (Peshenko et al., 2008; Zägel et al., 2013). The localization of all investigated mutants was very similar to the cellular localization of the WT. Using western blotting we further estimated that the amount of ROS-GC1 WT and mutants was nearly the same in a suspension of cells having a similar cell density (Figure 2B, also Zägel et al., 2013).

\section{BASAL GUANYLATE CYCLASE ACTIVITIES}

The mutant P575L exhibited similar basal GC activities as WT (Figure 3). Activities in the presence of GCAP1 and 2 at saturating $\left[\mathrm{Ca}^{2+}\right]$ revealed almost no difference between $\mathrm{P} 575 \mathrm{~L}$ and WT. However, a difference was observed, when GCAP1 and 2 were incubated with ROS-GC1 at low $\left[\mathrm{Ca}^{2+}\right.$ ] (EGTA columns in Figure 3) resulting in a $25-30 \%$ decrease of maximal activity.
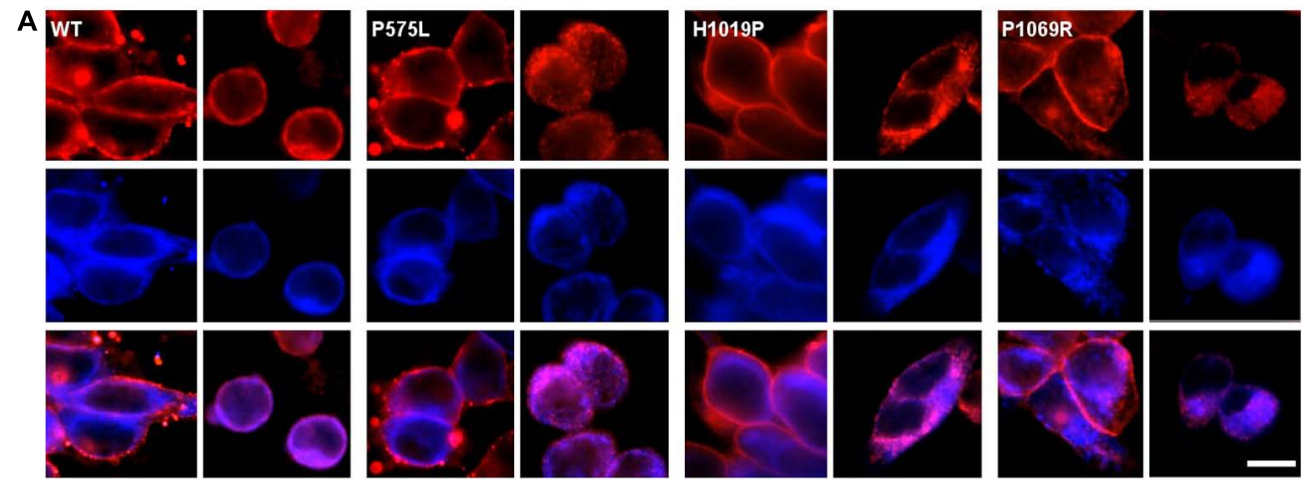

$150 \mathrm{kDa}$

B

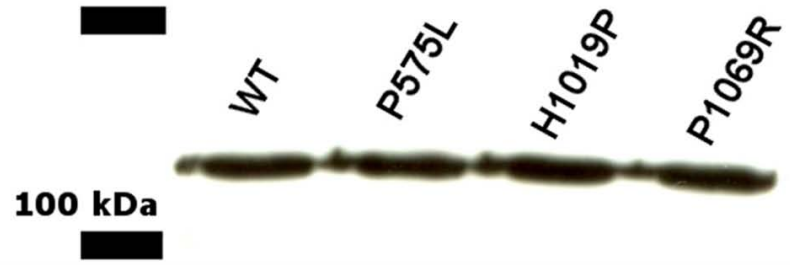

FIGURE 2 | Cellular localization ofWT and ROS-GC1 mutants in HEK293 cells. (A) Cells were stably transfected with human ROS-GC1 constructs and were probed with different antibodies; the corresponding ROS-GC1 construct used for transfection is indicated in the corner of the upper left panel.

Anti-Na ${ }^{+} / K^{+}$-ATPase was used as plasma membrane marker (upper left panel, red staining); anti-calnexin antibodies as marker for the endoplasmic reticulum (top right panel, red staining); anti-ROS-GC1 antibody (middle panels, blue staining); overlay of ROS-GC1 staining and membrane specific localization (lower panels). Secondary antibodies were indicated in the Methods section. Visualization was done in an Olympus fluorescence microscope. Cells were fixed either with paraformaldehyde (localization of plasma membrane) or with paraformaldehyde-methanol (endoplasmic reticulum). Scale bar $=10 \mu \mathrm{m}$. (B) Western blot of HEK293 cells expressing human ROS-GC1, WT and the mutants P575L, H1019P, and P1069R. Blots were probed with a primary antibody against ROS-GC1 (GC1\#3, dilution 1:2000) and a secondary anti-rabbit antibody from Dianova (dilution 1:4000) as described (Zägel et al., 2013). 


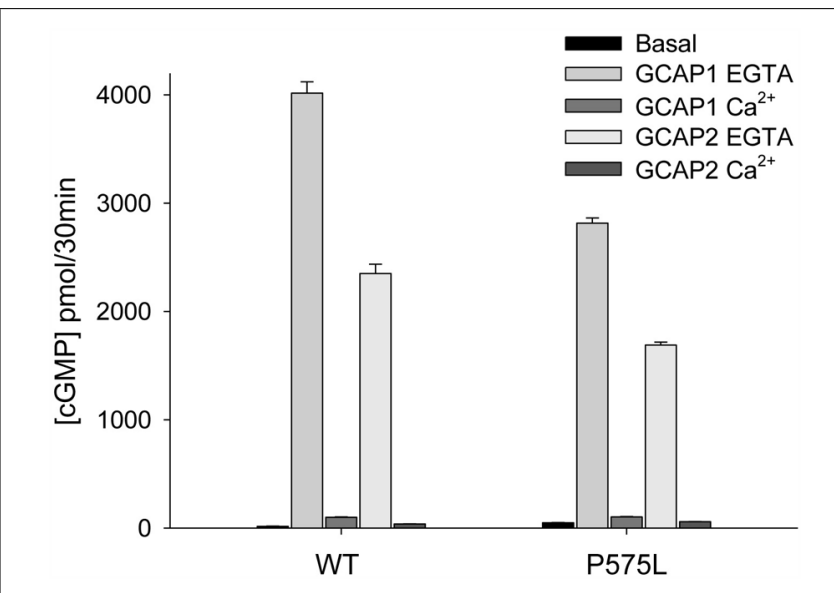

FIGURE 3 | Guanylate cyclase activities of WT and the P575L mutant. $\mathrm{Ca}^{2+}$-bound and $\mathrm{Ca}^{2+}$-free (EGTA) GCAP1 or GCAP2 were added to HEK293 cell membranes expressing human ROS-GC1 (inset) yielding a final concentration of $10 \mu \mathrm{M}$. $\mathrm{Ca}^{2}+$ was either at $33 \mu \mathrm{M}$ or $<10 \mathrm{nM}$ (EGTA). Assay incubations were performed with equal amounts of WT and mutant guanylate cyclase in membranes, which was verified by western blotting (see Figure 2B). Some error bars are not visible due to the scaling of the figure. Differences between data sets were highly significant yielding ***P $\leq 0.001$, except for P575L; when its basal activity was compared with the activity in the presence of $\mathrm{Ca}^{2+}$-bound GCAP2, it resulted in ${ }^{*} P \leq 0.01$.

In sharp contrast to P575L, the mutant P1069R did not exhibit any activity, neither in the presence nor in the absence of $\mathrm{Ca}^{2+}$, EGTA, or GCAPs (data not shown). We also tested activities of cells transiently expressing the mutant or a stably transfected cell line. Since the cellular localization of this mutant appeared normal and was very similar to the active WT and P575L forms (Figure 2A), we conclude that the lack of GC activity was a consequence of an impaired catalytic domain.

A special case was observed with the mutant H1019P: we were unable to obtain consistent activity profiles as we did with WT and the P575L mutant. In some cell batches we observed activation by GCAP1, but not by GCAP2, whereas in other batches both GCAPs were able to activate the cyclase or the enzyme was inactive under all conditions. The large scattering of the results seems to indicate a general instability of the mutant.

\section{GCAP-MEDIATED ACTIVATION}

The key feature among ROS-GC1 properties is the $\mathrm{Ca}^{2+}$ dependent activation under control of GCAPs. We tested this activation of P575L by GCAPs and compared it with the WT activity profile. Representative data sets are shown in Figure 4A (+GCAP1) and Figure 4B (+GCAP2), a summary of the $\mathrm{IC}_{50}$ values (mean $\pm \mathrm{SD}$ ) is displayed in Table 1. For both $\mathrm{Ca}^{2+}$-sensor proteins we observed a similar two-fold shift of the $\mathrm{IC}_{50}$ values to lower $\left[\mathrm{Ca}^{2+}\right]$, in the presence of GCAP1 from 0.529 (WT) to $0.270 \mu \mathrm{M}(\mathrm{P} 575 \mathrm{~L})$ and in the presence of GCAP2 from 0.269 $\mu$ (WT) to $0.128 \mu \mathrm{M}$ (P575L).

A shift in the $\mathrm{IC}_{50}$ values had previously been reported for some autosomal cone dystrophy related mutations that are located in a so-called "mutation hotspot" region within or near the residue R838 in the dimerization domain (Duda et al. 1999a, 2000; Wilkie

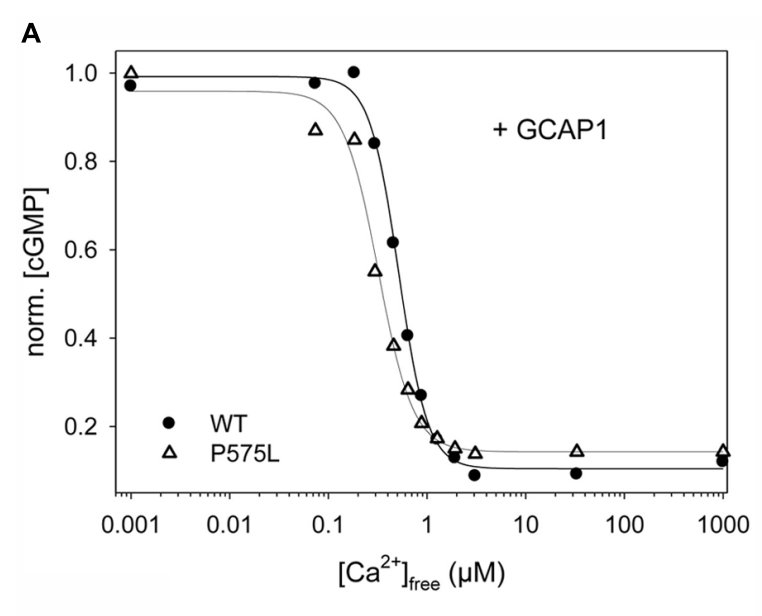

B

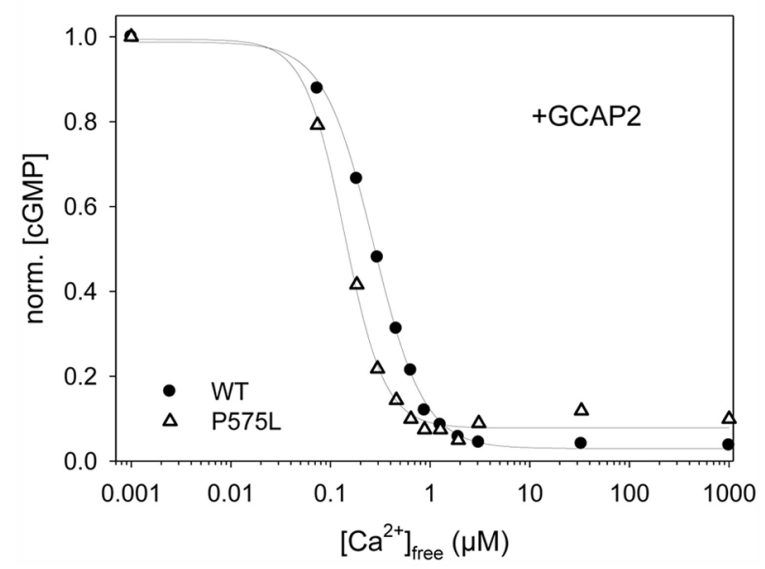

FIGURE $4 \mid \mathrm{Ca}^{2+}$-sensitive activity profiles of WT and ROS-GC1 mutant P575L. Guanylate cyclase activities were measured as a function of free $\left[\mathrm{Ca}^{2+}\right]$ for samples as indicated (A) WT or P575L, in the presence of $10 \mu \mathrm{M}$ GCAP1. (B) WT or P575L, in the presence of $10 \mu \mathrm{M}$ GCAP2. Incubation time was $30 \mathrm{~min}$. Activities are normalized to allow comparison of $I \mathrm{C}_{50}$ values $\left(\left[\mathrm{Ca}^{2+}\right]_{\text {free }}\right.$ at which activity is halfmaximal). Data are representative of three to four sets of incubations.

et al., 2000; Kitiratschky et al., 2008; Zägel et al., 2013). One reasonable model explaining this observed effect states that the shift in $\mathrm{Ca}^{2+}$-sensitivity originates from different apparent affinities of WT and mutant ROS-GC1 for the $\mathrm{Ca}^{2+}$-free form of GCAPs

Table 1 | Summary of $\mathrm{IC}_{50}$ and $\mathrm{EC}_{50}$ values for WT and P575L mutant.

\begin{tabular}{lrr}
\hline ROS-GC1 & \multicolumn{1}{c}{ IC $_{\mathbf{5 0}}(\boldsymbol{\mu} \mathbf{M})$} & \multicolumn{1}{c}{ EC $_{\mathbf{5 0}}(\boldsymbol{\mu} \mathbf{M})$} \\
\hline WT + GCAP1 & $0.529 \pm 0.006$ & $2.93 \pm 0.37$ \\
WT + GCAP2 & $0.269 \pm 0.009$ & $8.34^{+} \pm 0.36$ \\
P575L + GCAP1 & $0.270^{*} \pm 0.018$ & $3.32^{+} \pm 0.39$ \\
P575L + GCAP2 & $0.128 \pm 0.014$ & $7.44 \pm 1.26$
\end{tabular}

* Six data sets; ${ }^{+}$two data sets.

Values are the mean $\pm S D$ of mainly three to four independent measurements, if not indicated otherwise. 
A

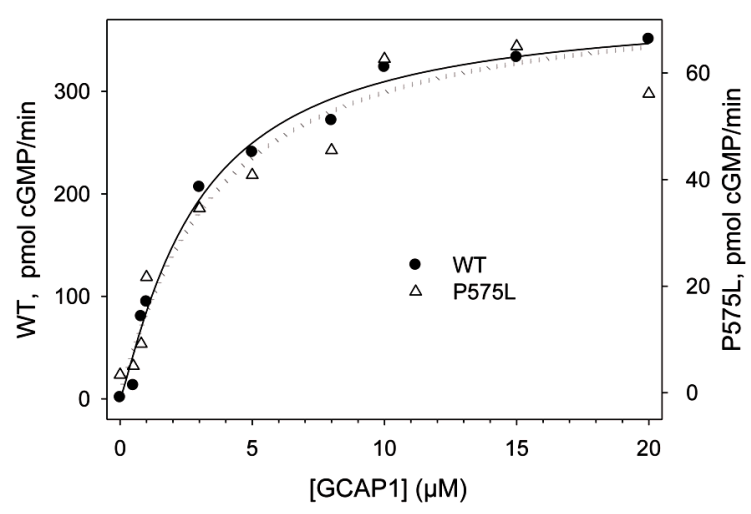

B

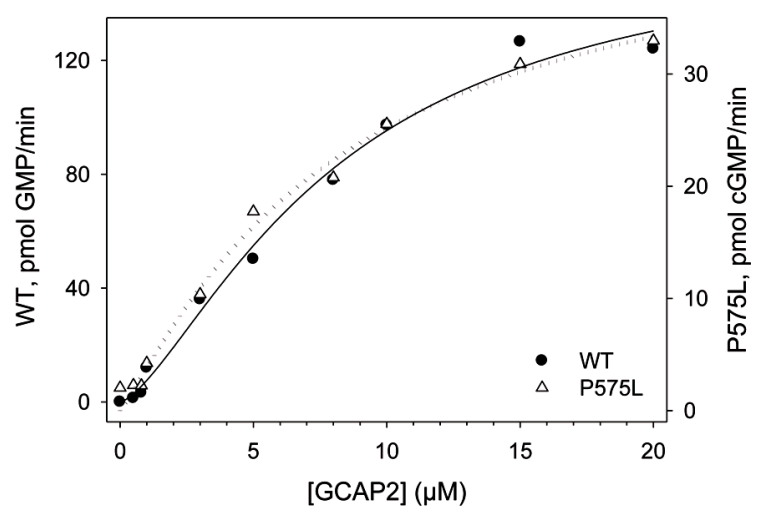

FIGURE 5 | GCAP-dependency of WT and P575L to determine apparent affinities $\left(E_{5_{00}}\right)$. WT and mutant P575L (see inset) were incubated with $\mathrm{Ca}^{2+}{ }_{- \text {free }}\left(\mathrm{Mg}^{2+}\right.$-bound) GCAP1 (A) and GCAP2 (B). The amount of cGMP produced by ROS-GC1 is shown as a function of the GCAP concentration. Incubation time was $30 \mathrm{~min}$.

(Peshenko et al., 2004). We tested this hypothesis for the P575L mutant by measuring the GC activities at increasing GCAP concentrations (Figure 5). GCAP1 and 2 were $\mathrm{Ca}^{2+}$-free, but they had $\mathrm{Mg}^{2+}$-bound (Peshenko and Dizhoor, 2004) under the assay conditions. Apparent affinities expressed as $\mathrm{EC}_{50}$-values differed only minimally between WT and the P575L mutant and overlapped within the measured standard deviations (Table 1). Thus, we conclude that differences in apparent affinities for GCAPs cannot account for the observed shifts in $\mathrm{Ca}^{2+}$-sensitivity of the P575L mutant.

\section{STRUCTURE-FUNCTION RELATIONSHIPS}

The single point mutation P575L is located in direct C-terminal neighborhood of a small stretch of 20 amino acids, which was previously identified as an essential regulatory and/ or interaction site in bovine ROS-GC1 for GCAP1 (L559-I578 corresponding to peptide \#34a in Lange et al., 1999). The region is highly conserved among different species and encompasses also the point mutation F565S (human sequence) that is linked to LCA type 1 (Perrault et al., 1996). However, the biochemical consequences of these two mutations differ significantly, since F565S leads to a more than 10-fold lower basal cyclase synthesis rate, but also to a complete loss of the GCAP1-stimulated GC activity (Duda et al., 1999b). These biochemical consequences are in contrast to the less severe effects caused by the P575L mutation.

The position H1019P is located in the short $\beta 6$-sheet of the CCD in close vicinity of the $\beta 5$-sheet that harbors amino acids R995, C997, L998, and F999, which are critical for binding the purine ring of the GTP substrate (Tucker et al., 1998). Replacement of $\mathrm{H}$ by $\mathrm{P}$ might cause a "kink" of the $\beta 6$-sheet and therefore disrupts or destabilize the catalytic center. A mutation of A to P in $\alpha$-synuclein for example was reported to have long-range and short-range effects on the three-dimensional structure including a decrease of $\beta$-sheet content (Wise-Scira et al., 2013). Furthermore, the position H1019P is also located in the GCAP2 binding site that was previously narrowed down to the amino acid stretch Y1012N1037 in the bovine sequence (Duda et al., 2005; Pettelkau et al., 2012). In summary, these results might explain the large scattering of GC activities observed in the in vitro assays.

In our test system the mutation P1069R had the most dramatic consequences leading to a completely inactive enzyme. This position is sandwiched between the binding sites of GCAP2 and S100 $\beta$ (Duda etal., 2002) and we can only speculate that it might have the function to integrate diverse signals. Furthermore, it seems to be indispensable for the basal catalysis of ROS-GC1.

\section{CONCLUSION}

Inherited retinal disorders can affect visual function in different manners. In the present study we investigated the biochemical consequences of three point mutations in the GUCY2D gene, which cause different forms of visual impairment. The mutation P575L causes a progressive form of cone degeneration in affected patients, which very often correlates with symptoms of photophobia, decrease of central vision and impairment of color vision. Since the mutation did not lead to a complete loss of guanylate cyclase function, cone cells expressing the mutated ROS-GC1 protein very likely maintain key features of photoreceptor physiology. But since the mutation induces a dysregulation of the $\mathrm{Ca}^{2+}$-sensitive cyclase activation profile, it seems to have longterm effects in the distortion of the $\mathrm{Ca}^{2+}{ }_{-}$GGMP homeostasis triggering apoptotic pathways. Similar consequences have been discussed for GCAP1 mutations correlating with certain forms of cone-rod dystrophies (Behnen et al., 2010; Hunt et al., 2010). The other two point mutations that were investigated in the present study correlate with LCA and a juvenile form of retinitis pigmentosa. Both mutations have more severe pathological consequences. Typical clinical features are for example an early onset of the disease in childhood and a strongly reduced or even nonrecordable electroretinogram (Perrault et al., 2000; Booji etal., 2005). Clinical manifestations of the point mutations appear to be mirrored in the biochemical properties of the expressed ROS$\mathrm{GC} 1$ protein as there are: synthesis of cGMP by ROS-GC1 is either severely disrupted due to protein instability (H1019P) or completely abolished (P1069R). Thus, light-triggered hydrolysis of cGMP cannot adequately be balanced by the second photoreceptor specific guanylate cyclase (ROS-GC2, GC-F), which has a much lower expression level, at least in cone cells (Olshevskaya 
et al., 2002; Baehr et al., 2007). The cGMP level in photoreceptor cells would be lower than in non-affected cell. This in turn would keep a lower number of cyclic nucleotide-gated channels open in the dark state. Since the cytoplasmic cGMP pool that is hydrolyzed after illumination is already reduced in the dark, amplitude and kinetics of the photoresponse are expected to be smaller and faster.

\section{ACKNOWLEDGMENT}

We thank Jutta Appelt for technical assistance in the cell culture laboratory and Dr. Alexander Scholten for fruitful discussions. This study was supported by a grant from the Deutsche Forschungsgemeinschaft (DFG) to Karl-Wilhelm Koch (KO948/10-1).

\section{REFERENCES}

Athanasiou, D., Aguila, M., Bevilacqua, D., Novoselov, S. S., Parfitt, D. A., and Cheetham, M. E. (2013). The cell stress machinery and retinal degeneration. FEBS Lett. 587, 2008-2017. doi: 10.1016/j.febslet.2013. 05.020

Baehr, W., Karan, S., Maeda, T., Luo, D.-G., Li, S., Bronson, J. D., et al. (2007). The function of guanylate cyclase $1(\mathrm{GC} 1)$ and guanylate cyclase $2(\mathrm{GC} 2)$ in rod and cone photoreceptors. J. Biol. Chem. 282, 8837-8847. doi: 10.1074/jbc.M6103 69200

Behnen, P., Dell'Orco, D., and Koch, K.-W. (2010). Involvement of the calcium sensor GCAP1 in hereditary cone dystrophies. Biol. Chem. 391, 631-637. doi: 10.1515/bc.2010.063

Booji, J. C., Florijn, R. J., ten Brink, J. B., Loves, W., Meire, F., von Schooneveld, M. J., et al. (2005). Identification of mutations in the AIPL1, CRB1, GUCY2D, RPE65, and RPGRIP1 genes in patients with juvenile retinitis pigmentosa. J. Med. Genet. 42, e67. doi: 10.1136/jmg.2005.035121

Boye, S. L., Peshenko, I. V., Huang, W. C., Min, S. H., McDoom, I., Kay, C. N., et al. (2013). AAV-mediated gene therapy in the guanylate cyclase (RetGC1/RetGC2) double knockout mouse model of Leber congenital amaurosis. Hum. Gene. Ther. 24, 189-202. doi: 10.1089/hum.2012.193

Buch, P. K., Mihelec, M., Cottrill, P., Wilkie, S. E., Pearson, R. A., Duran, Y., et al. (2011). Dominant cone-rod dystrophy: a mouse model generated by gene targeting of the GCAP1/Gucala gene. PLOS ONE 6:e18089. doi: 10.1371/journal.pone.0018089

den Hollander, A. I., Roepman, R., Koenekoop, R. K., and Cremers, F. P. M. (2008). Leber congenital amaurosis: genes, proteins and disease mechanisms. Prog. Retin. Eye Res. 27, 391-419. doi: 10.1016/j.preteyeres.2008.05.003

Dizhoor, A. M., Olshevskaya, E. V., and Peshenko, I. V. (2010). $\mathrm{Mg}^{2+} / \mathrm{Ca}^{2+}$ cation binding cycle of guanylyl cyclase activating proteins (GCAPs): role in regulation of photoreceptor guanylyl cyclase. Mol. Cell. Biochem. 334, 117-124. doi: 10.1007/s11010-009-0328-6

Duda, T., Fik-Rymarkiewicz, E., Venkataraman, V., Krishnan, R., Koch, K.-W., and Sharma, R. K. (2005). The calcium-sensor guanylate cyclase activating protein type 2-specific site in rod outer segment membrane guanylate cyclase type 1 . Biochemistry 44, 7336-7345. doi: 10.1021/bi050068x

Duda, T., Koch, K.-W., Venkataraman, V., Lange, C., Beyermann, M., and Sharma, R. K. (2002). $\mathrm{Ca}^{2+}$-sensor S100 modulated sites of membrane guanylate cyclase in the photoreceptor-bipolar synapse. EMBO J. 21, 2547-2556. doi: 10.1093/emboj/21.11.2547

Duda, T., Krishnan, A., Venkataraman, V., Lange, C., Koch, K.-W., and Sharma, R. K. (1999a). Mutations in the rod outer segment membrane guanylate cyclase (ROSGC1) in a cone-rod dystrophy cause defects in calcium signaling. Biochemistry 38, 13912-13919. doi: 10.1021/bi9915972

Duda, T., Venkataraman, V., Goraczniak, R., Lange, C., Koch, K.-W., and Sharma, R. K. (1999b). Functional consequences of a rod outer segment membrane guanylate cyclase (ROS-GC1) gene mutation linked with Leber's congenital amaurosis. Biochemistry 38, 509-515. doi: 10.1021/bi9824137

Duda, T., Venkataraman, V., Jankowska, A., Lange, C., Koch, K.-W., and Sharma, R. K. (2000). Impairment of the rod outer segment membrane guanylate cyclase dimerization in a cone-rod dystrophy results in defective calcium signaling. Biochemistry 39, 12522-12533. doi: 10.1021/bi001514d
Fain, G. L., Matthews, H. R., Cornwall, M. C., and Koutalos, Y. (2001). Adaptation in vertebrate photoreceptors. Physiol. Rev. 81, 117-151.

Hunt, D. M., Buch, P., and Michaelides, M. (2010). Guanylate cyclases and associated activator proteins in retinal disease. Mol. Cell. Biochem. 334, 157-168. doi: 10.1007/s11010-009-0331-y

Hwang, J.-Y., Lange, C., Helten, A., Höppner-Heitmann, D., Duda, T., Sharma, R. K., et al. (2003). Regulatory modes of rod outer segment membrane guanylate cyclase differ in catalytic efficiency and $\mathrm{Ca}^{2+}$-sensitivity. Eur. J. Biochem. 270, 3814-3821. doi: 10.1046/j.1432-1033.2003.03770.x

Jiang, L., Zhang, H., Dizhoor, A. M., Boye, S. E., Hauswirth, W. W., Frederick, J. M., et al. (2011). Long-term RNA interference gene therapy in a dominant retinitis pigmentosa mouse model. Proc. Natl. Acad. Sci. U.S.A. 108, 18476-18481. doi: 10.1073/pnas. 1112758108

Kaupp, U. B., and Seifert, R. (2002). Cyclic nucleotide-gated ion channels. Physiol. Rev. 82, 769-824.

Kitiratschky, V. B., Wilke, R., Renner, A. B., Kellner, U., Vadalà, M., Birch, D. G., et al. (2008). Mutation analysis identifies GUCY2D as the major gene responsible for autosomal dominant progressive cone degeneration. Invest. Ophthalmol. Vis. Sci. 49, 5015-5023. doi: 10.1167/iovs.08-1901

Koch, K.-W., and Dell'Orco, D. (2013). A calcium-relay mechanism in vertebrate phototransduction. ACS Chem. Neurosci. 4, 909-917. doi: 10.1021/cn400027z

Koch, K.-W., Duda, T., and Sharma, R. K. (2010). $\mathrm{Ca}^{2+}$-modulated vision-linked ROS-GC guanylate cyclase transduction machinery. Mol. Cell. Biochem. 334, 105-115. doi: 10.1007/s11010-009-0330-z

Koch, K.-W., and Helten, A. (2008). "Guanylate cyclase-based signaling in photoreceptors and retina," in Signal Transduction in the Retina, Chap. 6 eds S. J. Fliesler and O. G. Kisselev (North Tonawanda, NY: Taylor and Francis CRC Press), 121-143.

Krylov, D. M., Niemi, G. A., Dizhoor, A. M., and Hurley, J. B. (1999). Mapping sites in guanylyl cyclase activating protein-1 required for regulation of photoreceptor membrane guanylyl cyclases. J. Biol. Chem. 274, 10833-10839. doi: 10.1074/jbc.274.16.10833

Lange, C., Duda, T., Beyermann, M., Sharma, R. K., and Koch, K.-W. (1999). Regions in vertebrate photoreceptor guanylyl cyclase ROS-GC1 involved in $\mathrm{Ca}^{2+}$ dependent regulation by guanyly cyclase-activating protein GCAP-1. FEBS Lett. 460, 27-31. doi: 10.1016/S0014-5793(99)01312-5

Luo, D. G., Xue, T., and Yau, K.-W. (2008). How vision begins: an odyssey. Proc. Natl. Acad. Sci. U.S.A. 105, 9855-9862. doi: 10.1073/pnas.0708405105

Olshevskaya, E. V., Ermilov, A. N., and Dizhoor, A. M. (2002). Factors that affect regulation of cGMP synthesis in vertebrate photoreceptors and their genetic link to human retinal degeneration. Mol. Cell. Biochem. 230, 139-147. doi: 10.1023/A:1014248208584

Perrault, I., Rozet, J. M., Calvas, P., Gerber, S., Camuzat, A., Dollfus, H., et al. (1996). Retinal-specific guanylate cyclase gene mutations in leber's congenital amaurosis. Nat. Genet. 14, 461-464. doi: 10.1038/ng1296-461

Perrault, I., Rozet, J. M., Gerber, S., Ghazi, I., Ducroq, D., Souied, E., et al. (2000). Spectrum of retGC1 mutations in Leber's congenital amaurosis. Eur. J. Hum. Genet. 8, 578-582. doi: 10.1038/sj.ejhg.5200503

Peshenko, I. V., and Dizhoor, A. M. (2004). Guanylyl cyclase-activating proteins (GCAPs) are $\mathrm{Ca}^{2+} / \mathrm{Mg}^{2+}$ sensors: implications for photoreceptor guanylyl cyclase (RetGC) regulation in mammalian photoreceptors. J. Biol. Chem. 279, 1690316906. doi: 10.1074/jbc.C400065200

Peshenko, I. V., Moiseyev, G. P., Olshevskaya, E. V., and Dizhoor, A. M. (2004). Factors that determine $\mathrm{Ca}^{2+}$ sensitivity of photoreceptor guanylyl cyclase. Kinetic analysis of the interaction between the $\mathrm{Ca}^{2+}$-bound and the $\mathrm{Ca}^{2+}$-free guanylyl cyclase activating proteins (GCAPs) and recombinant photoreceptor guanylyl cyclase 1 (RetGC-1). Biochemistry 43, 13796-13804. doi: 10.1021/bi048943m

Peshenko, I. V., Olshevskaya, E., and Dizhoor, A. M. (2008). Binding of guanylyl cyclase activating protein 1 (GCAP1) to retinal guanylyl cyclase (RetGC1). J. Biol. Chem. 283, 21747-21757. doi: 10.1074/jbc.M801899200

Pettelkau, J., Schröder, T., Ihling, C. H., Olausson, B. E., Kölbel, K., Lange, C., et al. (2012). Structural insights into retinal guanylylcyclase-GCAP-2 interaction determined by cross-linking and mass spectrometry. Biochemistry 51, 4932-4949. doi: 10.1021/bi300064v

Pugh, E. N. Jr., and Lamb, T. D. (2000). "Phototransduction in vertebrate rods and cones: molecular mechanisms of amplification, recovery and light adaptation," in Handbook of Biological Physics, eds D. G. Stavenga, W. J. DeGrip, and E. N. Pugh Jr. (Leiden: Elsevier Science), 183-255. 
Ramamurthy, V., Tucker, C., Wilkie, S. E., Daggett, V., Hunt, D. M., and Hurley, J. B. (2001). Interactions within the coiled-coil domain of RetGC-1 guanylyl cyclase are optimized for regulation rather than for high affinity. J. Biol. Chem. 276, 26218-26229. doi: 10.1074/jbc.M010495200

Small, K. W., Silva-Garcia, R., Udar, N., Nguyen, E. V., and Heckenlively, J. R. (2008). New mutation, P575L, in the GUCY2D gene in a family with autosomal dominant progressive cone degeneration. Arch. Ophthalmol. 126, 397-403. doi: 10.1001/archopht.126.3.397

Stephen, R., Filipek, S., Palczewski, K., and Sousa, M. C. (2008). $\mathrm{Ca}^{2+}$-dependent regulation of phototransduction. Photochem. Photobiol. 84, 903-910. doi: 10.1111/j.1751-1097.2008.00323.x

Tucker, C. L., Hurley, J. H., Miller, T. R., and Hurley, J. B. (1998). Two amino acid substitutions convert a guanylyl cyclase, RetGC-1, into an adenylyl cyclase. Proc. Natl. Acad. Sci. U.S.A. 95, 5993-5997. doi: 10.1073/pnas.95. 11.5993

Wilkie, S. E., Newbold, R. J., Deery, E., Walker, C. E., Stinton, I., Ramamurthy, V., et al. (2000). Functional characterization of missense mutations at codon 838 in retinal guanylate cyclase correlates with disease severity in patients with autosomal dominant cone-roddystrophy. Hum. Mol. Genet. 9, 3065-3073. doi: 10.1093/hmg/9.20.3065

Wise-Scira, O., Aloglu, A. K., Dunn, A., Sakallioglu, I. T., and Coskuner, O. (2013). Structures and free energy landscapes of the wild-type and A30P mutant-type $\alpha$-synuclein proteins with dynamics. ACS Chem. Neurosci. 4, 486-497. doi: $10.1021 / \mathrm{cn} 300198 \mathrm{q}$

Zägel, P., Dell'Orco, D., and Koch, K.-W. (2013). The dimerization domain in outer segment guanylate cyclase is a $\mathrm{Ca}^{2+}$-sensitive control switch module. Biochemistry 52, 5065-5074. doi: 10.1021/bi400288p

Conflict of Interest Statement: The authors declare that the research was conducted in the absence of any commercial or financial relationships that could be construed as a potential conflict of interest.

Received: 25 November 2013; accepted: 10 February 2014; published online: 26 February 2014.

Citation: Zägel P and Koch K-W (2014) Dysfunction of outer segment guanylate cyclase caused by retinal disease related mutations. Front. Mol. Neurosci. 7:4. doi: 10.3389/fnmol.2014.00004

This article was submitted to the journal Frontiers in Molecular Neuroscience.

Copyright (c) 2014 Zägel and Koch. This is an open-access article distributed under the terms of the Creative Commons Attribution License (CC BY). The use, distribution or reproduction in other forums is permitted, provided the original author(s) or licensor are credited and that the original publication in this journal is cited, in accordance with accepted academic practice. No use, distribution or reproduction is permitted which does not comply with these terms. 